Experimental Support and Facilities Division Collider-Accelerator Department BROOKHAVEN NATIONAL LABORATORY Upton, New York 11973-5000

ES\&F Division Technical Note \# 168

Simulation of Soil Activation of Gold-Ions Hitting a Tungsten Target at J7 of AGS

K. Yip

December 1, 2010 


\title{
Simulation of soil activation of gold-ions hitting a tungsten target at $\mathrm{J} 7$ of AGS
}

\author{
December 1, 2010
}

\author{
Kin Yip \\ Experimental Support \& Facilities Division, Collider-Accelerator Department
}

\section{Introduction}

This time, the concern is the soil activation due to gold beam hitting a tungsten target of at J7. Simulations with MCNPX ${ }^{\mathrm{i}}$ with the gold ions of kinetic energy of $8.8648684 \times 197 \mathrm{GeV}$ or $1746379.07480 \mathrm{GeV}^{\mathrm{ii}}$ have been used to find the fluxes so that one may estimate the soil activation.

\section{Setup}

Figure 1 shows the cross-section of the geometry setup (perpendicular to the beam direction which is also the z-axis) in the MCNPX simulation. In the simulation, $y$-axis is pointing towards the sky and $\mathrm{x}$-axis is the remaining lateral dimension. The red area indicates the concrete wall which is of 1 foot thick. Outside the concrete wall, it's the soil (blue). In the simulation, I measure the fluxes of neutrons and protons inside the volume where $\mathrm{x}$ is between $10 \mathrm{~cm}$ and $20 \mathrm{~cm}$ (ie. a thickness of $10 \mathrm{~cm}$ ) behind the concrete wall (which is my typical way in soil activiation problems).

The tungsten target is 1 inch $\times 1$ inch with a thickness of 0.001 inches (or $2.54 \times 2.54 \times$

$\left.0.00254 \mathrm{~cm}^{3}\right)$ and the front face of the tungsten has the origin of the setup $(0,0,0)$, which is also where the pencil beam of gold ions hit. 


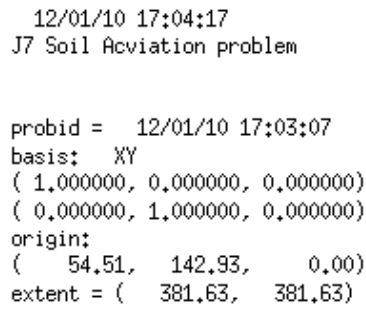

\begin{tabular}{|c|c|c|c|}
\hline \multirow[t]{2}{*}{ Edit } & \multirow[t]{2}{*}{ cel } & \multicolumn{2}{|l|}{2} \\
\hline & & & Cell \\
\hline$x y z=$ & 54.51 , & 142.93 & 0.00 \\
\hline CURSOR & & SCALES O & CellLine \\
\hline PostScript & & ROTATE & \\
\hline COLOR & mat & & \\
\hline$X Y$ & & $Y Z$ & $2 X$ \\
\hline LABEL & & sur & off \\
\hline
\end{tabular}

Click here or picture or menu

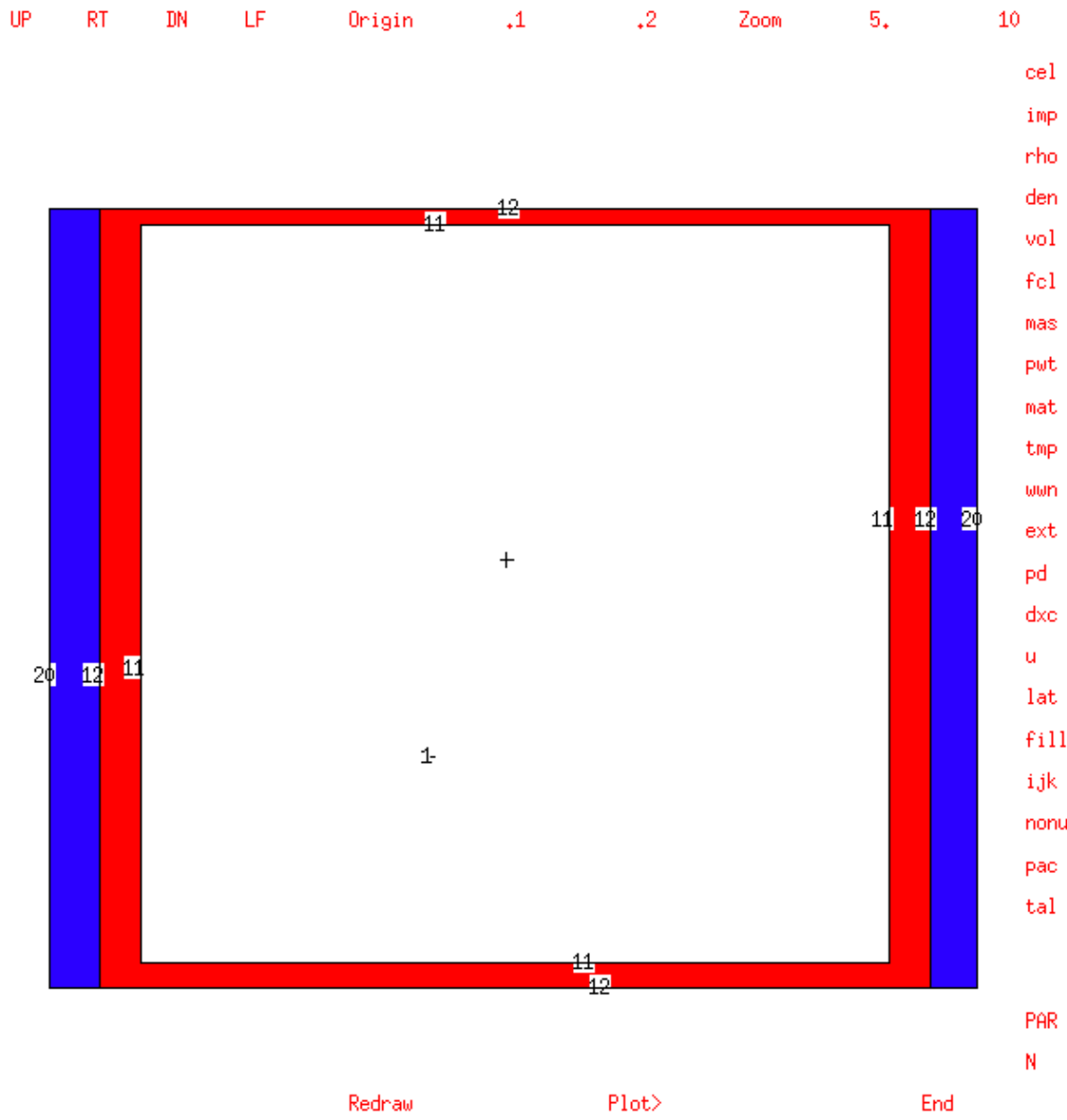

Figure 1: A picture of the MCNPX geometry used in the simulation. The blue area indicates the soil and the red area indicates the concrete wall. The place with the mark of " 1 " is where the tungsten target is.

\section{Results}

Figure 2 shows the neutron fluxes integrated over $\mathrm{x}$ from $10 \mathrm{~cm}$ to $20 \mathrm{~cm}$ in the $\mathrm{y}-\mathrm{z}$ plane in soil. After running over 130000000 events, the maximum neutron flux is $7.5181 \times 10^{-8}$ (statistical error of $\pm 3.86 \% \mathrm{~cm}^{-2}$ per incident gold ion and the maximum proton flux is $1.8334 \times 10^{-8}$ (statistical error of $\pm 7.92 \%$ ) $\mathrm{cm}^{-2}$ per incident gold. As they may not peak in the same place but conservatively, their sum gives a maximum flux is $\sim 9.351510^{-8} \mathrm{~cm}^{-2}$ per incident gold.

Assuming that the interaction length of $34 \mathrm{~cm}$ and the above flux, using the soil activation webpage calculators that I have written at http://www.cad.bnl.gov/kinyip/Radiation/Calculators.html, it would take about $9.2 \times 10^{16}$ gold ions to get to $1000 \mathrm{pCi} / \mathrm{L}$ of Tritium (which is $5 \%$ of the drinking water standard). 


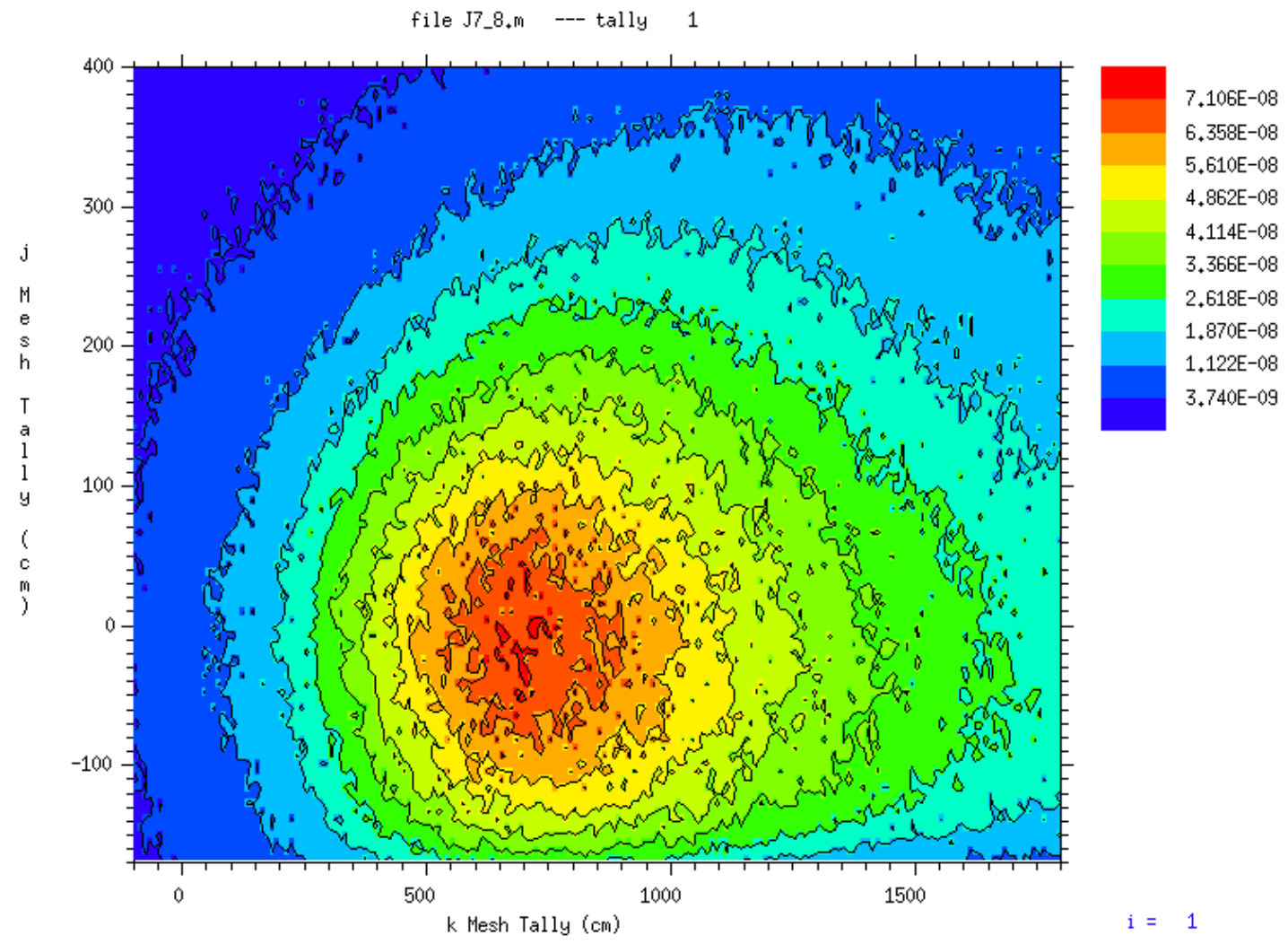

Figure 2: shows the neutron fluxes $\left(\mathrm{cm}^{-2}\right.$ per incident gold ion) in the $\mathrm{y}-\mathrm{z}$ plane in the soil of $\mathrm{x}$ from $10 \mathrm{~cm}$ to $20 \mathrm{~cm}$ behind the wall closer to the target. 


\section{Appendix}

The MCNPX input file is as follows :

Soil Acviation problem

c

c --- $(0,0,0)$ is the center of the Tungsten target

$\mathrm{c}$

c Tungsten target of inch/1000 thick

c

$13-19.30-1 \quad$ imp:e,n,p,h,\#,d,t,s,a=1

c

c vacuum inside tunnel

c

$201-11 \quad$ imp:e,n,p,h,\#,d,t,s,a=1

$\mathrm{c}$

c concrete wall

$\mathrm{c}$

$34-2.3511-12$ imp:e,n,p,h,\#,d,t,s,a=1

c

c the soil

c

$42-1.9 \quad 12-20 \quad$ imp:e,n,p,h,\#,d,t,s,a=1

c

$\mathrm{c}$

c -- don't care area

$99101220 \quad$ imp:e,n,p,h,\#,d,t,s,a=0

$\mathrm{c}===================================$

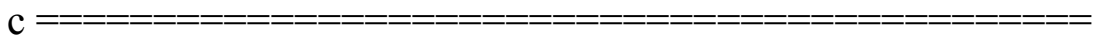

c

c Tungsten target of inch/1000 thick

$\mathrm{c}$

$\begin{array}{llllllll}1 \mathrm{rpp} & -1.27 & 1.27 & -1.27 & 1.27 & 0.0 .00254\end{array}$

c

c

c inner wall

c horizontal ( $7+11$ feet)

c floor to ceiling 17' 9"' (target 5' from floor)

c

$11 \mathrm{rpp} \quad-213.36 \quad 335.28 \quad-152.4 \quad 388.62 \quad-100.1900$.

c

c outer wall (including all concretes at the side but only a small portion on floor and ceiling) c

12 rpp $\quad-243.84 \quad 365.76 \quad-170.0 \quad 400.0 \quad-100.1900$. 


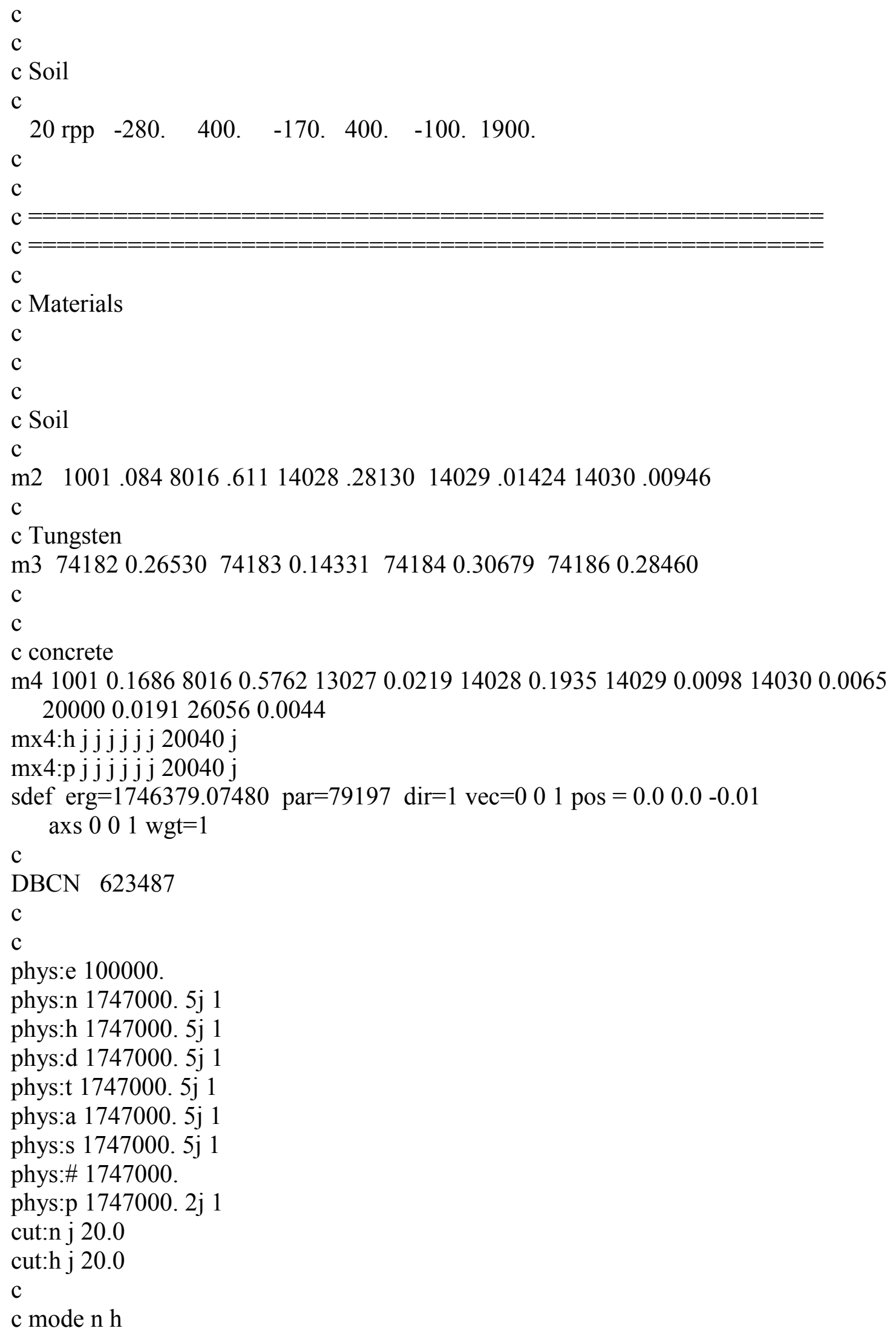


c

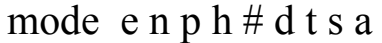

c

$\mathrm{c}$

c

nps 50000000

print

c

$\mathrm{c}$

prdmp $2 \mathrm{j} 1$

c

tmesh

rmesh 1:n flux

CORA1 -263.84 -253.84

CORB1 -170. 99i 400.

CORC1 -100. 189i 1800.

rmesh 11:h flux

CORA11 -263.84 -253.84

CORB11 -170. 99i 400.

CORC11 -100. 189i 1800.

rmesh21:n flux

CORA21 $375.76 \quad 385.76$

CORB21 -170. 99i 400.

CORC21 -100. 189i 1800.

rmesh31:h flux

CORA31 $375.76 \quad 385.76$

CORB31 -170. 99i 400.

CORC31 -100. 189i 1800.

endmd

${ }^{\mathrm{i}} \mathrm{MCNPX}$, version 2.7.c, http://menpx.lanl.gov.

ii The total and kinetic energies can be found in Section 7 on page 9 of the document written by Kip Gardner: http://www.cadops.bnl.gov/AGS/Operations/GardnerNotes/RhicRunParameters/barp10.pdf 